\title{
Evolución de la gravedad de la adicción a los dos años de tratamiento en pacientes heroinómanos
}

\author{
Gutiérrez, E.*; Sálz, P.A.**; García, N.*; Fernández, P.*; GonzÁlez, Mª.P**; Fernández, J.J.*; BobeS, J.** \\ * Servicios de Salud Mental del Principado de Asturias (SESPA). \\ * Área de Psiquiatría. Universidad de Oviedo.
}

Enviar correspondencia a:

Julio Bobes. Área de Psiquiatría. Facultad de Medicina. Julián Clavería, 6 - 3‥ 33006 Oviedo. Tel./Fax. 98510355 52. E-mail: frank@correo.uniovi.es

\section{RESUMEN}

Objetivos: Analizar la evolución de la gravedad de la dependencia, a los dos años, de una muestra de pacientes heroinómanos y averiguar el efecto que sobre dicha evolución tiene el hecho de mantenerse, o no, en contacto con el centro de tratamiento.

Pacientes y metodo: Durante septiembre 1998 - diciembre 1999 se ha procedido a la evaluación de una muestra compuesta por 100 pacientes heroinómanos que previamente (dos años antes) habían demandado tratamiento en la Unidad de Tratamiento de Toxicomanías de Mieres (Asturias). Evaluación: Protocolo "ad hoc", versión europea del Addiction Severity Index (EuropASI).

Resultados y conclusiones: En el momento de inclusión en el estudio, 31 pacientes fueron asignados a un programa de mantenimiento con metadona (PMM) y los 69 restantes, a tratamiento con naltrexona o a un programa libre de drogas (PLD). A los dos años, se localizaron 75 pacientes (3 fallecidos), por lo que se realizó el seguimiento a 72. En el momento basal, la gravedad de la adicción era bastante similar en los pacientes asignados a ambos tipos de programas (diferencias en el área médica, con mayor gravedad en los pacientes en PMM; $p=.017)$. A los dos años se constata que el hecho de haber recibido tratamiento y, sobre todo, el mantener contacto terapéutico con el centro disminuye la gravedad en la adicción en prácticamente todas las áreas a excepción de aquellas cuya evolución depende en menor medida del tratamiento específico de la dependencia: área médica y legal.

Palabras clave: Evolución, heroinómanos, gravedad de la adicción.

\section{INTRODUCCIÓN}

L a dependencia de opiáceos sigue siendo uno de los problemas de mayor magnitud dentro de los servicios sanitarios en nuestro país. El conocer el destino de estos pacientes en los diferentes tipos de tratamiento y como éstos influyen en las diferentes áreas de su vida, resulta fundamental para conseguir

\section{SUMMARY}

Objectives: To analyse the evolution of the severity of addiction (after two years) in a sample of heroin addicts and to discover the effect on the evolution of remaining in contact or not with the treatment centre.

Patients and Method: From September 1998 - December 1999, we evaluated 100 heroin addicts who had previously (two years before) requested treatment in a Drug Addiction Centre (Mieres - Asturias). The evaluation was made using an "ad hoc" protocol and the European version of the Addiction Severity Index (EuropASI).

Results and Conclusions: At baseline, 31 patients were assigned to a Methadone Maintenance Programme (MMP) and 69 patients to naltrexone treatment or a drug free program (DFP). After two years, 75 patients were contacted (3 had died). Follow-up was made with 72 . The severity of addiction at baseline was similar in those patients assigned to both programs (differences were only found in the medical area, greater severity in MMP; $p=.017$ ). After two years, we observed that those patients who had received treatment and had maintained contact with the centre had improved their severity of addiction in almost all areas except in those less related to the specific treatment of the dependence: medical and legal areas.

Key words: Follow-up, heroin addicts, severity of addiction. una mayor eficacia en las indicaciones terapéuticas, así como poder, en cierta medida, emitir un pronóstico a medio-largo plazo.

Son muchos los trabajos descriptivos que sobre drogodependencias se publican a lo largo del año. Sin embargo, los estudios de evolución son más escasos y difíciles en el campo de las drogodependencias, 
debido en gran parte a las dificultades que presentan este tipo de pacientes para mantenerse en contacto de forma regular con los servicios sanitarios (Grupo Interdisciplinar sobre Drogas, 1992) y a la elevada morbi-mortalidad que acompaña a la propia dependencia y enfermedades somáticas derivadas de ella (Vaillant, 1973; Stimsom et al., 1978; Haastrup y Jepsen, 1984, 1988). Además, la mayor parte de estos estudios no van más allá de dos años en la evaluación de resultados (Bobes et al, 1984; Melgarejo et al, 1986; Sánchez-Carbonell et al, 1986, 1988, 1989; García y Ezquiaga, 1992; San Narciso et al, 1998). Otras veces, los pacientes son reacios a facilitar información en los períodos de seguimiento, más aún si se encuentran fuera del programa de tratamiento (Sánchez-Carbonell et al, 1988; Marina, 1999).

En el presente estudio se pretende analizar la evolución a los dos años de una muestra de pacientes adictos a opiáceos, haciendo especial hincapié en la evolución de la gravedad de su adicción y en el efecto que sobre ésta tiene el mantenerse, o no, en contacto con el centro de tratamiento.

\section{PACIENTES Y MÉTODO}

\section{Pacientes}

La muestra se obtiene de los pacientes que consecutivamente demandaron tratamiento por su consumo de opiáceos en la Unidad de Tratamiento de Toxicomanías (U.T.T.) de Mieres (Area Sanitaria VII del Principado de Asturias, constituida por los concejos de Mieres, Aller y Lena), entre septiembre de 1996 y diciembre de 1997. Posteriormente se realiza un seguimiento de dichos pacientes a los dos años (septiembre de 1998 - diciembre 1999). La U.T.T. pertenece a los dispositivos de Salud Mental del Servicio de Salud del Principado de Asturias (S.E.S.P.A.) y atiende a los pacientes del sistema público, que con diagnóstico de drogodependencia solicitan tratamiento. El resto de dispositivos de Salud Mental lo componen una Unidad de Hospitalización Psiquiátrica, un Hospital de Día y un Centro de Salud Mental con dos departamentos, adultos e infantil. Los pacientes son atendidos por derivación desde el resto de servicios sanitarios del Area o por iniciativa propia.

\section{Criterios de inclusión}

Los criterios para ser incluidos en el presente estudio fueron los siguientes:

- Diagnóstico DSM-IV de dependencia de opiáceos.

- Ser mayor de 18 años.

- Proceso nuevo (solicitan consulta por primera vez en el centro) o paciente dado de alta previamente (el período de tiempo para considerar que un paciente fué dado de alta se establece en 6 meses sin acudir al centro).

- Consentimiento informado del paciente para la inclusión en el estudio.

\section{Criterios de exclusión}

Los criterios de exclusión han sido los siguientes:

- Politoxicómanos cuya principal droga de consumo no sea un opiáceo.

- Ser menor de 18 años.

\section{Evaluación realizada}

En todos los pacientes se investigaron variables sociodemográficas, de hábitos de consumo, legales, físicas, psíquicas, laborales, etcétera. De igual modo, se investigó la gravedad de la adicción mediante la versión europea del Addiction Severity Index (EuropASI; Kokkevi et al., 1995).

\section{Análisis estadístico}

En el análisis estadístico de las variables cuantitativas se utilizó el test t de Student para muestras independientes o la prueba $U$ de Mann-Whitney en las comparaciones intergrupo y el test $t$ de Student para datos apareados o la prueba W de Wilcoxon, para las comparaciones intragrupo. Para las variables cualitativas se utilizó la prueba chi-cuadrado (con corrección de Yates, siempre que fue posible, grados de libertad= 1) y el test exacto de Fisher. En todos los casos el nivel de significación estadística quedó establecido en el $5 \%$.

\section{RESULTADOS}

\section{Descripción basal de la muestra}

La muestra basal estaba compuesta por un total de 100 pacientes, de los cuales a 31 (31\%) se les indicó programa de mantenimiento con metadona (PMM), y a 69 se les realizó desintoxicación y posteriormente tratamiento con naltrexona o programa libre de drogas (PLD). La edad media de toda la muestra fué de 27.24 (5.54) años, siendo la de los pacientes en PMM de 30,71 (5.58) años y la de aquellos en PLD de $25.68(4.80)(p=.000)$. En cuanto al sexo, 85 pacientes eran varones y 15 mujeres, no encontrándose diferencias entre ambos tipos de tratamiento. El 73\% de los pacientes permanecían solteros y el 56\% mantienen la convivencia con la familia de origen. El nivel 
mayoritario (57\%) de estudios era de E.G.B. El 66\% habían realizado trabajos en los que no se requería ninguna formación, habitualmente en el sector de la minería, y en el momento de iniciar el tratamiento, el $25 \%$ se encontraban en situación de desempleo (Tabla 1). No se constataron diferencias estadísticamente significativas, en lo que a variables sociodemográficas se refiere, entre los pacientes asignados a las dos modalidades terapeúticas, PMM y PLD.

TABLA 1: Datos sociodemográficos.

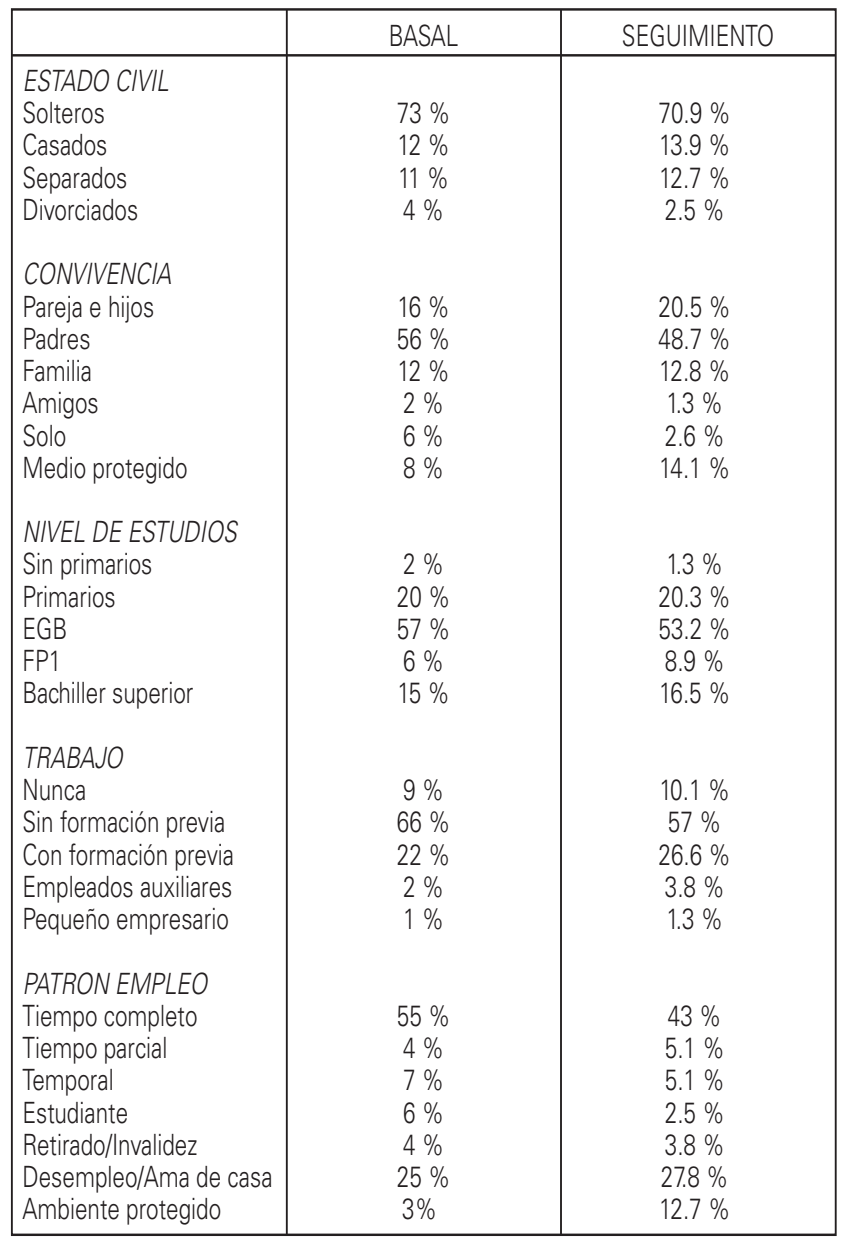

Del total de la muestra, 7 pacientes fueron seropositivos para el virus de la inmunodeficiencia humana (VIH); 42 habían padecido hepatitis por virus C y 26 hepatitis por el virus B. En los tres casos, la prevalencia de dichas infecciones víricas es mayor en los pacientes en PMM $(p=.000)$.

Las puntuaciones de gravedad basales obtenidas mediante el EuropASI son, en general, más elevadas en los pacientes en PMM (áreas médica, alcohol, drogas, legal y psicológica), siendo mayor solamente en los pacientes en PLD, en las áreas de soporte/empleo y familiar. No obstante, sólo existen diferencias estadísticamente significativas en el área médica, más elevada en los pacientes en PMM ( $p=$.017) (Tabla 2).
TABLA 2: Gravedad de la adicción según tratamiento indicado en el momento de inclusión en el estudio.

\begin{tabular}{|l|c|c|c|c|c|c|}
\hline \multirow{2}{*}{ Área EuropASI } & \multicolumn{3}{|c|}{ GRAVEDAD BASAL } & \multicolumn{3}{c|}{ GRAVEDAD A LOS 2 AÑOS } \\
\cline { 2 - 8 } & $\begin{array}{c}\text { PLD (n=69) } \\
\text { Media (SD) }\end{array}$ & $\begin{array}{c}\text { PMM (n=31) } \\
\text { Media (SD) }\end{array}$ & $P^{*}$ & PLD (n=47) & PMM (n=25) & $p^{*}$ \\
& $1.43(1.34)$ & $2.52(2.23)$ & .017 & $2.02(1.82)$ & $3.84(2.56)$ & .003 \\
\hline Médica & $4.74(2.27)$ & $4.55(2.69)$ & NS & $3.74(2.61)$ & $3.48(2.28)$ & NS \\
\hline Empleo & $1.72(0.91)$ & $1.97(1.05)$ & NS & $1.26(0.67)$ & $1.24(0.83)$ & NS \\
\hline Alcohol & $6.93(0.83)$ & $7.23(0.76)$ & NS & $4.74(2.50)$ & $5.04(2.24))$ & NS \\
\hline Drogas & $2.20(2.00)$ & $3.03(2.46)$ & NS & $2.17(2.06)$ & $3.44(2.57)$ & .039 \\
\hline Legal & $4.52(2.09)$ & $3.74(2.37)$ & NS & $3.96(2.69)$ & $3.60(2.42)$ & NS \\
\hline Familia & $2.62(2.17)$ & $2.74(2.44)$ & NS & $2.00(1.64)$ & $1.80(1.32)$ & NS \\
\hline Psíquica & & & & & & \\
\hline
\end{tabular}

* Prueba t de Student (datos independientes).

\section{Descripción de la muestra a los dos años}

A los dos años de iniciado el tratamiento se ha podido localizar a un total de 75 pacientes $(63$ hombres y 12 mujeres; $75 \%$ de la muestra basal), de los cuales 3, todos ellos hombres, habían fallecido. Por tanto, a la hora de realizar el seguimiento se ha podido contar con 72 pacientes, concretamente con 47 (68.12\%) pacientes de los 69 que inicialmente fueron asignados a un PLD y con 25 (80.64\%) de los 31 que inicialmente se enrolaron en un PMM.

En la Tabla 3 se muestran las puntuaciones basales de gravedad de la adicción de los 72 pacientes que pudieron ser localizados a los dos años y de los 28 que no fueron localizados. De igual modo, se muestra idéntica comparación en función del tratamiento basal asignado (PLD o PMM). Como puede observarse en dicha tabla, no se observaron diferencias estadísticamente significativas en ninguna de las áreas del EuropASI, por lo que puede considerarse que la gravedad de la adicción en el momento de inclusión en el estudio de los pacientes localizados y los no localizados era similar. Tampoco se constataron diferencias estadísticamente significativas en las variables sociodemográficas, clínicas, toxicológicas y legales entre los pacientes que pudieron ser localizados a los dos años y los que no.

De los pacientes a los que se consiguió realizar el seguimiento a los 2 años, el $70.9 \%$ permanecen solteros, el $13.9 \%$ casados y el $15.2 \%$ separados o divorciados. Un $48.7 \%$ viven con sus padres y un $14.1 \%$ solos. El 53.2\% tienen estudios de E.G.B., el 20.3\% primarios y el $16.5 \%$ bachiller superior. Un $57 \%$ han trabajado en labores que no requerían formación previa y un $26.6 \%$ con formación anterior, y la mayoría (34\%) a tiempo completo (Tabla 1). 
TABLA 3: diferencias en la gravedad de la adicción basal entre pacientes con seguimiento a los dos años y pacientes sin seguimiento.

\begin{tabular}{|c|c|c|c|c|c|c|c|c|c|}
\hline \multirow[t]{2}{*}{ Área EuropASI } & \multicolumn{3}{|c|}{ Total pacientes $(n=100)$} & \multicolumn{3}{|c|}{ PMM en momento basal $(n=31)$} & \multicolumn{3}{|c|}{ PLD en momento basal $(n=69)$} \\
\hline & $\begin{array}{l}\text { Localizados* } \\
(n=72) \\
\text { Media (SD) }\end{array}$ & $\begin{array}{l}\text { No Localiz** } \\
\text { (n=28) } \\
\text { Media (SD) }\end{array}$ & $p^{a}$ & $\begin{array}{c}\text { Localizados* } \\
(n=25) \\
\text { Media (SD) }\end{array}$ & $\begin{array}{l}\text { No Localiz** } \\
\quad(n=6) \\
\text { Media (SD) }\end{array}$ & $p^{b}$ & $\begin{array}{l}\text { Localizados* } \\
\quad(n=47) \\
\text { Media (SD) }\end{array}$ & $\begin{array}{l}\text { No Localiz** } \\
(n=22) \\
\text { Media (SD) }\end{array}$ & $p^{a}$ \\
\hline Médica & $1.83(1.78)$ & 1.61 (1.62) & NS & $2.52(2.14)$ & $2.50(2.81)$ & NS & $1.47(1.46)$ & $1.36(1.09)$ & NS \\
\hline Empleo & $4.68(2.41)$ & $4.68(2.40)$ & NS & $4.72(2.62)$ & $3.83(3.31)$ & NS & $4.66(2.32)$ & $4.91(2.20)$ & NS \\
\hline Alcohol & $1.82(0.88)$ & $1.75(1.14)$ & NS & $1.80(0.50)$ & $2.67(2.16)$ & NS & $1.83(1.03)$ & $1.50(0.51)$ & NS \\
\hline Drogas & $7.07(0.81)$ & $6.89(0.83)$ & NS & $7.12(0.78)$ & $7.67(0.52)$ & NS & $7.04(0.83)$ & $6.68(0.78))$ & NS \\
\hline Legal & $2.32(2.07)$ & $2.82(2.42)$ & NS & $2.88(2.37)$ & $3.67(2.94)$ & NS & $2.02(1.85)$ & $2.59(2.28)$ & NS \\
\hline Familia & $4.18(2.23)$ & $4.54(2.12)$ & NS & $3.72(2.42)$ & $3.83(2.42)$ & NS & $4.43(2.11)$ & $4.73(2.07)$ & NS \\
\hline Psíquica & $2.81(2.37)$ & $2.29(1.86)$ & NS & $2.88(2.62)$ & $2.17(1.32)$ & NS & $2.77(2.26)$ & $2.32(1.99)$ & NS \\
\hline
\end{tabular}

* Pacientes que a los dos años pudieron ser localizados y a los que se realizó el seguimiento.

* Pacientes que a los dos años no pudieron ser localizados.

${ }^{\text {a }}$ Prueba t de Student (datos independientes).

bPrueba U de Mann-Whitney (datos independientes).

Los pacientes que inicialmente fueron asignados a un PLD, obtuvieron a los dos años puntuaciones más elevadas de gravedad que los inicialmente asignados a un PMM, en las áreas de empleo/soportes, alcohol, familia y psicológica, si bien es necesario señalar que dichas diferencias no resultaron estadísticamente significativas. Por el contrario, los pacientes que inicialmente fueron asignados a un PMM, puntuaron, a los dos años, más que los inicialmente asignados a un PLD, en las areas médica $(p=.003)$ y legal $(p=.039)$ (Tabla 2).

En la Tabla 4 se muestra la situación de los pacientes a los dos años de iniciarse el estudio. De los 69 que inicialmente fueron asignados a un PLD, un $11.6 \%$ estaban en PMN, un $15.9 \%$ habían pasado a un PMM, un $1.4 \%$ a comunidad terapeútica (CT), un $21.7 \%$ no estaban en ningún tipo de tratamiento pero se mantenían abstinentes de consumir opiáceos, un $17.4 \%$ no seguían a tratamiento y consumían opiáceos, un $2.9 \%$ habían fallecido y, finalmente, un $29.0 \%$ estaban en situación desconocida. De los 31 inicialmente asignados a PMM, un $54.8 \%$ seguían en idéntica situación a los dos años, un $9.7 \%$ habían pasado a $\mathrm{CT}$, un $6.4 \%$ no estaban a tratamiento pero se mantenían abstinentes a opiáceos, un 9.7\% habían dejado el tratamiento y habían vuelto a consumir opiáceos, un $3.2 \%$ habían fallecido y, por último, un $16.1 \%$ estaban en paradero desconociso. Como puede observarse en dicha tabla, no se observan diferencias estadísticamente significativas en el porcentaje, a los dos años, de personas abstinentes de opiáceos, consumidores de opiáceos, fallecidos o personas en paradero desconocido en función del tratamiento basal asignado.
TABLA 4: Programa terapéutico a los dos años.

\begin{tabular}{|l|c|c|c|}
\hline Tratamiento a los dos años & $\begin{array}{c}\text { Tratamiento basal } \\
\text { PLD (n=69) }\end{array}$ & $\begin{array}{c}\text { Tratamiento basal } \\
\text { PMM }(n=31)\end{array}$ & $P^{*}$ \\
\hline PMN & $8(11.6 \%)$ & & \\
PMM & $11(15.9 \%)$ & $17(54.8 \%)$ & \\
CT & $1(1.4 \%)$ & $3(9.7 \%)$ & \\
No tratamiento + En abstinencia & $15(21.7 \%)$ & $2(6.4 \%)$ & NS \\
No tratamiento + Consumiendo & $12(17.4 \%)$ & $3(9.7 \%)$ & NS \\
Fallecidos & $2(2.9 \%)$ & $1(3.2 \%)$ & NS \\
Situación desconocida & $20(29.0 \%)$ & $5(16.1 \%)$ & NS \\
\hline
\end{tabular}

$P M N=$ Programa de mantenimiento con maltrexona. PMM= Programa de mantenimiento con metadona. CT= Comunidad terapéutica.

* Prueba chi-cuadrado con corrección de Yates.

\section{Evolución de la historia toxicológica}

Observando la historia toxicológica de los pacientes se puede apreciar que el $100 \%$ han probado en alguna ocasión el tabaco, alcohol, la heroína y los derivados del cannabis (Tabla 5). Los consumos basales y actuales de las diferentes sustancias se expresan en la Tabla 5. Como puede observarse en dicha tabla se ha experimentado un descenso significativo $(p<.05)$ del porcentaje de consumidores de cannabis y de LSD, mientras que el resto de consumos permanecen estables.

En cuanto la vía de administración de la heroína, del $44 \%$ que utilizaban la intravenosa en el momento 
TABLA 5: Historia toxicológica.

\begin{tabular}{|l|c|c|c|c|}
\hline DROGA & $\begin{array}{c}\text { Consumo } \\
\text { alguna vez (\%) }\end{array}$ & $\begin{array}{c}\text { Edad Inicio } \\
\text { Media (SD) }\end{array}$ & $\begin{array}{c}\text { Consumo basal (\%) } \\
n=100\end{array}$ & $\begin{array}{c}\text { Consumo actual (\%) } \\
n=72\end{array}$ \\
\hline Tabaco & 100 & $13.78(2.76)$ & 99 & 98.7 \\
\hline Alcohol & 100 & $14.9(1.94)$ & 65 & 64.1 \\
\hline Heroina & 100 & $20.1(4.32)$ & 100 & 36.4 \\
\hline BDZ & 91 & $22(5.21)$ & 69 & 37.7 \\
\hline Cocaina & 96 & $19.57(3.78)$ & 35 & 20.8 \\
\hline Anfetaminas & 71 & $17.89(3.03)$ & 5 & 0 \\
\hline Extasis & 42 & $20.43(4.06)$ & 13 & 0 \\
\hline Cannabis* & 100 & $15.92(2.36)$ & 37 & 24.7 \\
\hline LSD* $^{*}$ & 72 & $17.67(2.99)$ & 6 & 3.2 \\
\hline
\end{tabular}

* Diferencias estadísticamente significativas entre porcentaje de consumidores basal y actual (prueba chi-cuadrado con corrección de Yates)

$\mathrm{BZD}=$ Benzodiacepinas

basal, se ha pasado a un $35.7 \%$ a los dos años, aumentando por tanto el uso de la vía respiratoria en ese período entre los que aún consumen dicha droga. Igual ocurre con las benzodiacepinas que han variado de un $18.8 \%$ de la vía respiratoria en la observación basal al 3.6\%, aumentando la proporción de la vía oral. Sin embargo, con la cocaína ocurre lo contrario, ya que en el estudio basal el consumo por vía intravenosa suponía un $32.4 \%$ frente a los dos años que era de un $37.5 \%$, en detrimento de la vía respiratoria (55.9\% vs. $50 \%)$. La cantidad media de heroína consumida al inicio del estudio era de $603.75 \mathrm{mg}$ (495.58) frente a a los $167.21 \mathrm{mg}$ (324.83) consumidos a los dos años. Aquellos pacientes a los que se consiguió realizar el seguimiento, habían iniciado el consumo de benzodiacepinas a los 22.49 (5.39) años frente a los que no se logró reevaluar a los dos años que era de 19.53 (3.38) años $(p=.012)$. Igual ocurre con la edad de inicio del consumo de cocaína [19.95 (3.89) vs 17.69 (2.47) ( $p=$ .005)].

\section{Evolución de la gravedad de la dependencia}

Como ya se ha comentado, a los dos años, conseguimos entrevistar de forma comprehensiva (administración del EuropASI) a un total de 72 pacientes (72\% de la muestra inicial). Al analizar la evolución de la gravedad de su dependencia, se observan mejorías significativas $(p<.05)$ en prácticamente todas las áreas, a excepción de la relacionada con problemas médicos (existe un deterioro significativo, $p=.002$ ), del área referida a problemas legales, en la que se detecta un deterioro no significativo y del área familiar y social en la que se detecta una mejoría no significativa (Tabla 6).

Cuando se analizan el total de pacientes que a los dos años permanecen en contacto con el centro de tratamiento $(n=40)$, se observan resultados totalmente superponibles a los obtenidos con la muestra total (Tabla 6).

El análisis de los 32 pacientes que pudieron ser reevaluados a los dos años, pero que habían cesado el contacto con el centro de tratamiento, pone de manifiesto que éstos sólo experimentaron mejorías significativas en las áreas de alcohol $(p=.000)$ y drogas $(p=$ .000), no observándose variaciones en el resto de las áreas (Tabla 6).

TABLA 6: Evolución a los dos años de la gravedad de la adicción.

\begin{tabular}{|c|c|c|c|c|c|c|c|c|c|}
\hline \multirow{2}{*}{$\begin{array}{l}\text { Área } \\
\text { EuropASI }\end{array}$} & \multicolumn{3}{|c|}{ Muestra total $(n=72)$} & \multicolumn{3}{|c|}{ Pacientes en contacto* $(n=40)$} & \multicolumn{3}{|c|}{ Pacientes sin contacto ${ }^{*} \mid(n=32)$} \\
\hline & $\begin{array}{c}1^{a} \text { visita } \\
\text { Media (SD) }\end{array}$ & $\begin{array}{c}2^{\text {a }} \text { visita } \\
\text { Media (SD) }\end{array}$ & $p^{a}$ & $\begin{array}{c}1^{\mathrm{a}} \text { visita } \\
\text { Media (SD) }\end{array}$ & $\begin{array}{c}2^{\mathrm{a}} \text { visita } \\
\text { Media (SD) }\end{array}$ & $p^{a}$ & $\begin{array}{c}1^{\text {a }} \text { visita } \\
\text { Media (SD) }\end{array}$ & $\begin{array}{c}2^{\text {a }} \text { visita } \\
\text { Media (SD) }\end{array}$ & $p^{b}$ \\
\hline Médica & $1.83(1.78)$ & $2.65(2.27)$ & .002 & $1.80(1.67)$ & $2.80(2.33)$ & .009 & $1.88(1.95)$ & $2.47(2.20)$ & NS \\
\hline Empleo & $4.68(2.41)$ & $3.65(2.48)$ & .000 & $4.63(2.37)$ & $3.55(2.45)$ & .002 & $4.75(2.50)$ & $3.78(2.56)$ & NS \\
\hline Alcohol & $1.82(0.88)$ & $1.25(0.73)$ & .000 & $1.83(0.98)$ & $1.30(0.88)$ & .002 & $1.81(0.74)$ & $1.19(0.47)$ & .000 \\
\hline Drogas & $7.07(0.81)$ & $4.85(2.40)$ & .000 & $6.95(0.81)$ & $5.08(1.80)$ & .000 & $7.22(0.79)$ & $4.56(2.99))$ & .000 \\
\hline Legal & $2.32(2.07)$ & $2.61(2.31)$ & NS & $2.55(2.32)$ & $3.08(2.52)$ & NS & $2.03(1.69)$ & $2.03(1.91)$ & NS \\
\hline Familia & $4.18(2.23)$ & $3.83(2.58)$ & NS & $3.58(2.12)$ & $3.45(2.29)$ & NS & $4.94(2.17)$ & $4.31(2.88)$ & NS \\
\hline Psíquica & $2.81(2.37)$ & $1.93(1.53)$ & .000 & $2.58(2.18)$ & $1.50(0.91)$ & .002 & $3.09(2.59)$ & $2.47(1.95)$ & NS \\
\hline
\end{tabular}

* Pacientes que a los dos años permanecían en contacto con los dispositivos asitenciales.

* Pacientes que a los dos años habían perdido el contacto con los dispositivos asistenciales.

aprueba t de Student (datos apareados).

brueba W de Wilcoxon (datos apareados). 
Del grupo de pacientes a los que se indicó tratamiento en PMM, 25 (80.64\% de los 31 iniciales), pudieron volver a ser evaluados comprehensivamente a los dos años, observándose descensos significativos en las puntuaciones de gravedad de las áreas de empleo/soportes $(p=.008)$, alcohol $(p=.002)$, drogas $(p=.001)$ y psicológica $(p=.024)$. Tal y como ocurría cuando se consideraba toda la muestra, independientemente del tratamiento basal asignado, se observó un deterioro significativo del área médica $(p=.035)$ (Tabla 7).

La evolución de la gravedad de la dependencia de los pacientes incluídos basalmente en PMM y que continúan en contacto a los dos años con el centro de tratamiento $(n=20)$, es similar a la descrita anteriormente (Tabla 7).

Los pacientes asignados inicialmente a PMM que, a pesar de no permanecer en contacto con el centro, pudieron ser reevaluados fueron tan sólo 5 , lo cual es un número muy bajo para poder extraer conclusiones. No obstante, y de modo orientativo, la evolución de sus puntuaciones se muestra en la Tabla 7, no detectándose variaciones en ninguna de las áreas del EuropASI.

Los pacientes incluídos en PLD que pudieron ser reevaluados a los dos años fueron 47 (69.12\% de los 69 iniciales). Dichos pacientes evolucionaron de modo similar a los incluidos en PMM (Tabla 8).

TABLA 7: Evolución a los dos años de la gravedad de la adicción en los pacientes originariamente en PMM

\begin{tabular}{|c|c|c|c|c|c|c|c|c|c|}
\hline \multirow{2}{*}{$\begin{array}{l}\text { Área } \\
\text { EuropASI }\end{array}$} & \multicolumn{3}{|c|}{ Total PMM (n=25) } & \multicolumn{3}{|c|}{ Pacientes en contacto* $(n=20)$} & \multicolumn{3}{|c|}{ Pacientes sin contacto** $(n=5)$} \\
\hline & $\begin{array}{c}1^{\mathrm{a}} \text { visita } \\
\text { Media (SD) }\end{array}$ & $\begin{array}{c}2^{\mathrm{a}} \text { visita } \\
\text { Media (SD) }\end{array}$ & $p^{a}$ & $\begin{array}{c}1^{\text {a }} \text { visita } \\
\text { Media (SD) }\end{array}$ & $\begin{array}{c}2^{\mathrm{a}} \text { visita } \\
\text { Media (SD) }\end{array}$ & $p^{a}$ & $\begin{array}{c}1^{\mathrm{a}} \text { visita } \\
\text { Media (SD }\end{array}$ & $\begin{array}{c}2^{\mathrm{a}} \text { visita } \\
\text { Media (SD }\end{array}$ & $p^{a}$ \\
\hline Médica & $2.52(2.23)$ & $3.84(2.56)$ & .035 & $2.29(2.03)$ & $3.75(2.49)$ & .033 & $4.00(3.03)$ & $4.20(3.11)$ & NS \\
\hline Empleo & $4.55(2.69)$ & $3.48(2.28)$ & .008 & $4.29(2.63)$ & $3.50(2.19)$ & .023 & $5.00(3.16)$ & $3.40(2.88)$ & NS \\
\hline Alcohol & $1.97(1.05)$ & $1.24(0.83)$ & .002 & $1.86(0.48)$ & $1.25(0.91)$ & .005 & $1.67(0.52)$ & $1.20(0.45)$ & NS \\
\hline Drogas & $7.23(0.76)$ & $5.04(2.24)$ & .001 & $7.05(0.80)$ & $5.00(1.97)$ & .001 & $7.50(0.55)$ & $5.20(3.42))$ & NS \\
\hline Legal & $3.03(2.46)$ & $3.44(2.57)$ & NS & $3.05(2.54)$ & $3.55(2.58)$ & NS & $2.67(2.25)$ & $3.00(2.74)$ & NS \\
\hline Familia & $3.74(2.37)$ & $3.60(2.42)$ & NS & $3.38(2.20)$ & $3.25(2.12)$ & NS & $5.17(2.93)$ & $5.00(3.24)$ & NS \\
\hline Psíquica & $2.74(2.44)$ & $1.80(1.32)$ & .024 & $2.62(2.50)$ & $1.50(0.76)$ & .046 & 3.33 (2.88) & $3.00(2.35)$ & NS \\
\hline
\end{tabular}

* Pacientes que a los dos años permanecían en contacto con los dispositivos asitenciales.

** Pacientes que a los dos años habían perdido el contacto con los dispositivos asistenciales.

a Prueba W de Wilcoxon (datos apareados).

TABLA 8: Evolución a los dos años de la gravedad de la adicción en los pacientes originariamente en PLD.

\begin{tabular}{|c|c|c|c|c|c|c|c|c|c|}
\hline \multirow{2}{*}{$\begin{array}{l}\text { Área } \\
\text { EuropASI }\end{array}$} & \multicolumn{3}{|c|}{ Total PMM $(n=47)$} & \multicolumn{3}{|c|}{ Pacientes en contacto* $(n=20)$} & \multicolumn{3}{|c|}{ Pacientes sin contacto ${ }^{*}(n=27)$} \\
\hline & $\begin{array}{c}1^{\mathrm{a}} \text { visita } \\
\text { Media (SD) }\end{array}$ & $\begin{array}{c}2^{\mathrm{a}} \text { visita } \\
\text { Media (SD) }\end{array}$ & $p^{a}$ & $\begin{array}{c}1^{\mathrm{a}} \text { visita } \\
\text { Media (SD) }\end{array}$ & $\begin{array}{c}2^{a} \text { visita } \\
\text { Media (SD) }\end{array}$ & $p^{b}$ & $\begin{array}{c}1^{a} \text { visita } \\
\text { Media (SD) }\end{array}$ & $\begin{array}{c}2^{a} \text { visita } \\
\text { Media (SD) }\end{array}$ & $p^{b}$ \\
\hline Médica & $1.47(1.46)$ & $2.02(1.82)$ & .038 & $1.25(0.85)$ & $1.85(1.76)$ & NS & $1.59(1.70)$ & $2.15(1.90)$ & .038 \\
\hline Empleo & $4.66(2.32)$ & $3.74(2.61)$ & .013 & $4.79(2.25)$ & $3.60(2.74)$ & .037 & $4.72(2.46)$ & $3.85(2.55)$ & NS \\
\hline Alcohol & $1.83(1.03)$ & $1.26(0.67)$ & .000 & $1.67(1.24)$ & $1.35(0.88)$ & NS & $1.83(0.76)$ & $1.19(0.48)$ & .000 \\
\hline Drogas & $7.04(0.83)$ & $4.74(2.50)$ & .000 & $6.79(0.83)$ & $5.15(1.66)$ & .000 & $7.17(0.80)$ & $4.44(2.97)$ & .001 \\
\hline Legal & $2.02(1.85)$ & $2.17(2.06)$ & NS & $2.29(2.29)$ & $2.60(2.41)$ & NS & $2.03(1.64)$ & $1.85(1.73)$ & NS \\
\hline Familia & $4.43(2.11)$ & $3.96(2.69)$ & NS & $4.08(2.22)$ & $3.65(2.48)$ & NS & $4.83(2.00)$ & $4.19(2.86)$ & NS \\
\hline Psíquica & $2.77(2.26)$ & $2.00(1.64)$ & .009 & 2.58 (1.98) & $1.50(1.05)$ & .028 & $3.03(2.54)$ & $2.37(1.90)$ & NS \\
\hline
\end{tabular}

* Pacientes que a los dos años permanecían en contacto con los dispositivos asitenciales.

* * Pacientes que a los dos años habían perdido el contacto con los dispositivos asistenciales.

a Prueba t de Student (datos apareados).

a Prueba W de Wilcoxon (datos apareados). 
Cuando se consideran sólo a aquellos pacientes a los que se indicó hace dos años un PLD y que continúan en contacto con el centro al realizar el seguimiento $(n=20)$, se observan mejorías en las áreas de empleo/soportes $(p=.037)$, drogas $(p=.000)$ y psicológica $(p=.000)$. Cabe reseñar que el hecho de no encontrar una mejoría significativa en el área de alcohol no debe de extrañarnos, puesto que estos pacientes obtenían puntuaciones de gravedad basales muy bajas en dicha área (Tabla 8).

Por último, de los pacientes incluídos inicialmente en PLD, que no continúan en contacto con el centro, se consiguió reevaluar a 27. Dichos pacientes mejoran en las áreas de alcohol $(p=.000)$ y drogas $(p=.001)$ y empeoran en el área médica $(\mathrm{p}=.038)$ (Tabla 8).

\section{DISCUSION}

La muestra de pacientes ha sido extraída de una de las Areas Sanitarias más castigadas por la drogadicción en Asturias, y en la que el desempleo y la recesión económica han tenido más incidencia en los últimos años. Su homogeneidad sociodemográfica viene facilitada por la existencia de dispositivos únicos de Salud Mental y por la concentración en un sólo centro, de la atención a las toxicomanías.

\section{Resultados basales}

\section{Datos sociodemográficos}

En lo referente a los aspectos sociodemográficos, estos pacientes responden a las características observadas, en general, en otros estudios. Así, la edad de los pacientes se corresponde con uno de los tramos donde la prevalencia de la adicción a opiáceos es más abundante (25 a 29 años) y mantiene una relación por sexos muy similar a la que presentan la mayoría de autores (Amodia, 1992; Comas et al, 1992; Marina, 1996; P.R.D., 1994; S.E.I.T., 1995; Ruiz et al, 1996; Cervera et al, 1997; Gutiérrez et al, 1998; San Narciso et al, 1998).

En cuanto al estado civil, predominan los pacientes solteros. Datos similares se encuentran en trabajos realizados con pacientes de entornos similares (Amodia et al, 1.992; Marina, 1.996; Cervera et al, 1997; San Narciso et al, 1998). En los trabajos de Gutiérrez et al (1996) y Ruiz et al (1996), el porcentaje de pacientes solteros es mayor (72\% y $84 \%$ respectivamente).

En lo referente a la convivencia, los resultados obtenidos tampoco difieren de una forma importante con los obtenidos por otros autores. Así, predomina la convivencia con los padres, sobre todo los varones solteros, siendo más frecuente en el sexo femenino, la convivencia en pareja o en solitario (Amodia, 1992; Comas, 1992; Gomez-Zapata, 1992; Marina 1996).
El nivel de instrucción de los pacientes se acerca más a los resultados obtenidos por Marina (1996) y San Narciso et al (1998), que a los de otros autores (Amodia, 1992; Ruiz 1996). El resultado era esperable, si tenemos en cuenta que los primeros autores han trabajado con pacientes de la misma Comunidad Autónoma.

\section{Variables toxicológicas y clínicas.}

En el presente estudio, la hepatitis C, se presenta como la infección vírica más prevalente en el colectivo de drogodependientes. Pese a ser la infección vírica más prevalente en éste colectivo, es ligeramente menor en el presente estudio que en otros trabajos consultados (Lozano et al, 1996; Herrera et al, 1997) y mayor que en el presentado por San Narciso et al (1998).

Como en el resto de nuestra Comunidad Autónoma, la infección por el VIH es de las más bajas de nuestro entorno. En el presente estudio, la prevalencia de seropositivos para el VIH es muy escasa; posiblemente sea debido a la restricción impuesta por los criterios de inclusión en el estudio, ya que al aceptar solamente a los pacientes que demandaban tratamiento, se estaba excluyendo a aquellos que estaban incluídos anteriormente en PMM, dónde la frecuencia de VIH positivos, y la retención en el tratamiento es mayor, por lo que es un dato poco valorable. Aún así, son datos muy inferiores a los presentados por Cervera et al (1997) y más en la línea de los obtenidos por San Narciso et al (1998).

Al igual que en otros estudios (Amodia, 1992), la mayoría de los pacientes atendidos en el período del estudio, han sido incluídos en PMN.

La mayor gravedad y, por tanto, de necesidad de tratamiento de los pacientes, se encuentra en el área de drogas y en el familiar y de empleo/soportes. Los tres resultados son lógicos, a la vista de los problemas derivados de la toxicomanía, con aumento de la problemática familiar y el dato, no exclusivo de esta población, del paro y la escasa formación académica, que dificulta el encuentro de un trabajo estable (San Narciso et al, 1998).

\section{Seguimiento a los dos años}

\section{Pacientes reclutados}

Se ha conseguido localizar y entrevistar a los dos años de iniciado el estudio al $72 \%$ de los pacientes. Las dificultades para lograr mayores porcentajes de pacientes residen en el frecuente cambio de domicilio de este tipo de pacientes, períodos de encarcelamiento, ingresos en Comunidades Terapéuticas que en ocasiones no se encuentran en la misma provincia, por no olvidar aquellos con una especial problemática 
social y de marginalidad que les lleva en ocasiones a no poseer un domicilio (Marina, 1996). Los estudios de seguimiento localizan, en general a un número elevado de pacientes, aunque consiguen entrevistar a porcentajes mucho mas reducidos de los mismos (Stimson et al, 1978; Marina, 1996). Por este motivo creemos que en el presente estudio el número de pacientes entrevistados ha sido importante aunque solamente han trascurrido dos años de la valoración basal. El resto de los estudios consultados difieren en el número de años a los que se realiza la evaluación, aunque, en general, también presentan porcentajes importantes de reevaluaciones (Stimson et al, 1978; Guardia et al, 1983, 1987; Haastrup et al, 1984, 1988; Sánchez-Carbonell et al, 1989; Marina, 1996; Landabaso et al, 1996; Jiménez et al, en prensa).

\section{Variables sociodemográficas}

El estado civil de los pacientes ha evolucionado de forma natural, disminuyendo el número de solteros y aumentando el de casados y separados. Marina (1996) encuentra valores parecidos aunque los porcentajes son más elevados en ambos casos. Algo similar ocurre con la convivencia y el nivel de estudios, aunque en nuestro estudio el nivel de instrucción es mayor. Marina (1996) y Melús y Gutiérrez (1996) presentan tasas de paro más elevadas que las nuestras, además, en nuestro estudio se observa, en líneas generales, una mejoría del patrón de empleo a los dos años.

\section{Variables toxicológicas}

Se aprecia una importante mejoría en lo que a consumos de sustancias se refiere a excepción del tabaco y el alcohol. Salvo en el caso de heroína, no podemos saber si han aumentado o no la cantidad consumida, ya que solamente valoramos si existe consumo actual o no. De todas formas, solamente es significativo el descenso de consumidores en el caso del cannabis y LSD. Melús y Gutiérrez (1996), Marina (1996) y Fernández Miranda et al (1999) aprecian, como en nuestro caso, un descenso en el consumo de sustancias psicoactivas en los pacientes que permanecen en tratamiento.

\section{Evolución de la gravedad de la adicción.}

A la vista de nuestros resultados, podemos decir que, en líneas generales, la evolución de nuestra muestra ha sido positiva, no observándose prácticamente diferencias de evolución en función del tratamiento basal indicado, ya que aunque los pacientes asignados a PMM presentan una mayor gravedad a los dos años en las áreas médica y legal, también partían de puntuaciones iniciales más elevadas en dichas áreas, si bien las diferencias basales sólo eran significativas en el caso del área médica.
Por otra parte, la evolución de los pacientes en PMM que siguen en contacto con el centro, pone de manifiesto mejoría en cuatro áreas (empleo/soportes, alcohol, drogas y psicológica) y deterioro en el área médica. Idénticas apreciaciones pueden realizarse en los pacientes enrolados en PLD que siguen en contacto con el centro, si bien, en éstos no se observa un deterioro en el área médica (ya se ha comentado que partían de una situación basal más favorable en esta área) y tampoco se observa mejoría en el área de alcohol, aunque cabe señalar que a tenor de las puntuaciones de gravedad basales obtenidas en dicha área, el alcohol no constuía un problema relevante en estos pacientes.

Cuando se tiene en cuenta la evolución de los pacientes que a los dos años no seguían en contacto con el centro de tratamiento, los resultados obtenidos son más pobres, ya que globalmente sólo se observan mejorías en las áreas de alcohol y drogas. Este dato puede venir a ilustrar la importancia de la retención terapeútica y de los tratamientos a largo plazo a la hora de obtener una mejoría global del paciente adicto a opiáceos.

En los diferentes estudios consultados se evalúa la gravedad desde diferentes parámetros, la mayoría indirectos (consumo de sustancias, situación laboral, problemática legal, relaciones familiares...), siendo muy pocos los que utilizan instrumentos standard, lo que hace dificil la comparación. Pese a todo parece existir un sentir unánime entre todos los autores a la hora de valorar los cambios producidos en los dependientes de opiáceos que se someten a tratamiento. Así, Melús y Gutiérrez (1996) observan dicha mejoría a la vez que señalan el menor uso de otros recursos terapéuticos cuando los pacientes permanecen mas tiempo en tratamiento. Landabaso et al (1996) aprecian en sus pacientes una mejoría global de estos en función del mayor tiempo de permanencia en contacto con los centros de tratamiento. Arias et al (1996) observan una mejoría en todas las áreas del ASI, excepto la médica que permanece igual y la de consumo de alcohol que empeora en función de la permanencia en tratamiento. Zannini et al (1997) afirman que los pacientes que permanecen en tratamiento tienden a llevar un curso favorable en su dependencia, mejorando las relaciones familiares y amistosas. De igual forma, San Narciso et al (1998) señalan una mejoría en todas las áreas del EuropASI en los pacientes que permanecen en tratamiento, aunque los resultados solamente son significativos en el área familiar y social. Por último, Fernández et al (1999) aprecian una mejoría en todos los aspectos de la vida de los pacientes que permanecen en contacto con el centro.

Al igual que en los trabajos comentados, nuestros pacientes mejoran en casi todas las áreas del EuropASI, a excepción de la médica. Esta circunstancia pueden ser debida, por una parte, a la mayor concien- 
ciación del cuidado que precisan sus patologías orgánicas y por otra al empeoramiento lógico que sucede en enfermedades crónicas como puede ser la hepatitis por virus $\mathrm{C}$ y el VIH. El hecho de que no se constaten mejorías en el área legal, podría atribuirse a la lentitud con que se resuelven los procesos legales en nuestro país, lo que lleva a que pacientes ya estabilizados en diferentes aspectos de su adicción comiencen a recibir sentencias del pasado o estén a punto de ser sometidos a juicios de épocas anteriores. Por último, cabe señalar que la mejoría detectada en el área familiar y social no es significativa, posiblemente debido a que es dificil hacer virar en un espacio de tiempo relativamente corto la gran problemática familiar y social que se deriva de la toxicomanía.

A la vista de los resultados se puede afirmar que el recibir tratamiento $y$, sobre todo, el mantener el contacto terapéutico con el centro de tratamiento se asocian con una mejoría en la gravedad de la adicción de los pacientes con dependencia a opiáceos en prácticamente todas las áreas a excepción de aquellas cuya evolución depende en menor medida del tratamiento específico de la dependencia; nos referimos a las áreas médica (cronicidad de las enfermedades relacionadas con el consumo de sustancias), el área relacionada con los aspectos legales (tardanza en la resolución de los procesos judiciales, lo que lleva a tener que cumplir sentencias estando en el programa terapéutico) y el área familiar y social (enquistamiento de problemas familiares y sociales). También es posible que los pacientes busquen tratamiento precisamente por la seguridad de que sea atenuante a la hora de cumplir las sentencias que se pueden derivar de juicios pendientes.

\section{CONCLUSIONES}

1. Iniciar tratamiento se asocia con una disminución de la gravedad de diferentes áreas relacionadas con la dependencia, independientemente del tipo de tratamiento indicado

2. El permanecer en contacto con el centro de tratamiento se asocia, en general, a una mejoría de la gravedad de la adicción en los pacientes dependientes de opiáceos

\section{BIBLIOGRAFÍA}

Amodia Díez R (1992): Epidemiología de las toxicomanías en la Red de Salud Mental de Asturias (1980-1989). Cuadernos Asturianos de Salud. Oviedo, Servicio de Publicaciones del Principado de Asturias.

Arias F, López-lbor JJ, Ochoa E (1996): Predictores evolutivos en un programa de mantenimiento con Naltrexona. Adicciones, 8 (4): 479-500.
Bobes J, Bousoño M, Fernández JM, et al (1984): Evaluación prospectiva de 473 toxicodependientes en Asturias (1979-1982). Actas Luso-Esp Neurol Psiquiatr, 12 (5): 347-356

Cervera G, Valderrama JC, Bolinches F, et al (1997): Pauta de desintoxicación frente a estabilización y mantenimiento con metadona en adictos a opiáceos con trastorno de personalidad. Psiquiatría Biológica, 4 (5): 181-186.

Comas D, Carrón J, Hernández MI, et al (1992): Metodología de los estudios de seguimiento. Resultados de una investigación. Adicciones, 4 (2): 111-126.

Delegación del Gobierno para el Plan Nacional sobre Drogas (1996): Sistema estatal de información sobre toxicomanías (S.E.I.T.) 1.995. Madrid, Ministerio de Justicia e Interior. pp 43-50.

Fernández JJ, González MP, Sáiz P, et al (1999): Calidad de vida y severidad de la adicción en heroinómanos en mantenimiento prolongado con metadona. Adicciones, 11 (1): 43-52.

García A, Ezquiaga E (1992): Estudio de seguimiento a seis meses de una muestra de drogodependientes. Arch Neurobiol, 55 (5): 215-223.

Gómez-Zapata M, Osuna E, Seiquer C, et al (1992): Patología orgánica asociada en adictos a drogas por vía parenteral. Estudio en una muestra de 200 pacientes. Adicciones, 4 (2): 127-134.

Grupo Interdisciplinar sobre Drogas (1992): Los estudios de seguimiento. Una experiencia metodológica. Madrid, Editorial Fundamentos.

Gutiérrez E, Domper J, Iglesias CA (1996): Características de pacientes drogodependientes según el orden en el consumo de heroína. Libro de Actas de las XXIII Jornadas Nacionales de Socidrogalcohol. Oviedo. pp 531-533.

Gutiérrez E, Sáiz PA, González MP, et al (1998): Definición del perfil de buen respondedor a los programas de mantenimiento con naltrexona. Addiciones, 10 (4): 321-333.

Haastrup S, Jepsen P (1984): Seven years follow-up of 300 young drug abusers. Acta Psychiatr Scand, 70 (5): 503-509.

Haastrup S, Jepsen P (1988): Eleven year follow-up of 300 young opioid addicts. Acta Psychiatr Scand, 77 (1): 22-26.

Herrera Ballesteros A (1997): Hepatopatías en drogodependencias. Libro de Actas de las XXIV Jornadas Nacionales de Socidrogalcohol. Alicante. pp 487-492.

Jiménez L, Sáiz PA, Gutiérrez E, et al (en prensa): Valoración transversal tras quince años en una muestra de adictos a opiáceos en Asturias. Adicciones.

Kokkevi A, Hartgers C (1995): EuropASI: European adaptation of a multidimensional assessment instrument for drug and alcohol dependence. Eur Addict Res, 1: 208210.

Landabaso MA, Fernández B, Sanz J, et al (1996): Estudio de la evolución (1990-1993) de adictos a opiáceos en programa de antagonistas. Adicciones, 8 (1): 67-74. 
Lozano JL, Vada J, Izaguirre M, et al (1996): Complicaciones médicas asociadas al consumo de drogas. Estudio en una U.D.H.. Libro de Actas de las XXIII Jornadas Nacionales de Socidrogalcohol. Oviedo. pp 567-570.

Marina P (1996): Adictos a la heroína en Asturias. Un estudio de seguimiento. En: Bobes J, González-Quiros P, González MP (eds.), 30 Años de alcohol y otras drogas en Asturias.Oviedo, Gofer.

Marina P (1999): Qué nos han enseñado los estudios de seguimiento de toxicómanos. Adicciones, 11 (3): 237241.

Melgarejo M, Sanahuja P, Massferrer J, et al (1986): Estudio de seguimiento en población heroinómana. Fons Informatiu, 1: 1-38.

Melús R, Gutiérrez T (1996): Programa de mantenimiento con naltrexona: seguimiento a los 12 meses. Adicciones, 8 (1): 5- 18.

Plan Regional sobre Drogas (1995): Memoria 1.994. Oviedo, Servicio de Publicaciones del Principado de Asturias.

Ruiz R, Casete L, Carrera IH, et al (1996): Estudio preliminar sobre la incidencia y valor pronóstico del codiagnóstico psiquiátrico en usuarios de una Comunidad Terapeútica. Libro de Actas de las XXIII Jornadas Nacionales de Socidrogalcohol. Oviedo. pp 591-596.
San Narciso Gl, Carreño JE, Pérez SF, et al (1998): Evolución de los trastornos de personalidad evaluados mediante el IPDE en una muestra de pacientes heroinómanos en tratamiento con naltrexona. Adicciones, 10 (1): 7-22.

Sánchez-Carbonell J, Camí J (1986): Recuperación de heroinómanos: definición, criterios y problemas de los estudios de evaluación y seguimiento. Medicina Clínica, 87 (9): 377-382.

Sánchez-Carbonell J, Camí J, Brigos B (1988): Follow-up of heroin addicts in Spain (EMETYST Project): results 1 year after treatment admission. Br J Addict, 83 (12): 1439-1448.

Sánchez-Carbonell J, Camí J, Brigos B (1989): Evolución de una muestra de heroinómanos dos años después del inicio del tratamiento (proyecto EMETYST). Medicina Clínica, 4: 135-139.

Stimson G, Oppenheimer E, Thorley A (1978): Seven years follow-up of heroin addicts: drug use and outcome. BMJ, 1: 1190-1192.

Vaillant GE (1973): A twenty-year follow-up of New York narcotic addicts. Arch Gen Psychiatry, 29: 237-241.

Zannini A, Nizzoli U, Bussolati N (1997): Valoración y seguimiento de los tratamientos con metadona: índice de retención, marcadores biológicos y comportamentales. Adicciones, 9 (3): 457-466. 